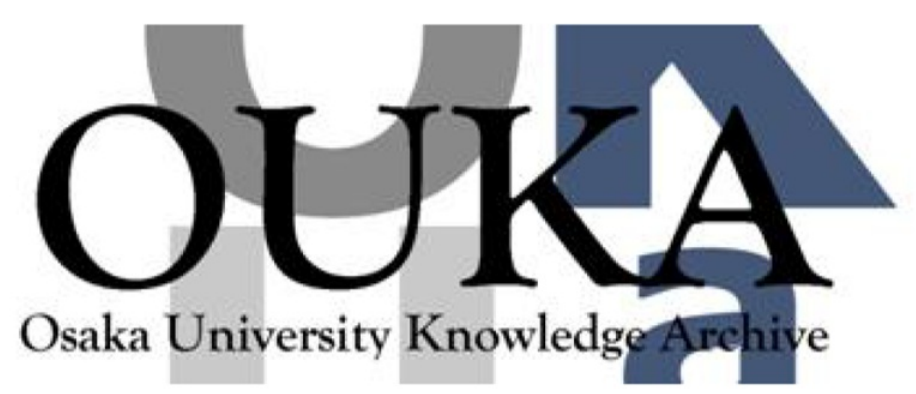

\begin{tabular}{|c|c|}
\hline Title & $\begin{array}{l}\text { Replacement-free mass-amplified sandwich assay } \\
\text { with } 180-\mathrm{MHz} \text { electrodeless quartz-crystal } \\
\text { microbalance biosensor }\end{array}$ \\
\hline Author (s) & $\begin{array}{l}\text { Ogi, Hirotsugu; Yanagida, Taiji; Hirao, } \\
\text { Masahiko et al. }\end{array}$ \\
\hline Citation & $\begin{array}{l}\text { Biosensors and Bioelectronics. 26(12) p. 4819- } \\
\text { p. } 4822\end{array}$ \\
\hline Issue Date & $2011-05-30$ \\
\hline oaire:version & AM \\
\hline URL & https://hdl. handle. net/11094/84194 \\
\hline rights & $\begin{array}{l}\text { (- } 2011 \text { Elsevier B.V. This manuscript version is } \\
\text { made avai lable under the Creative Commons } \\
\text { Attribut ion-NonCommercial-NoDerivatives } 4.0 \\
\text { International License. }\end{array}$ \\
\hline Note & \\
\hline
\end{tabular}

Osaka University Knowledge Archive : OUKA

https://ir. Library. osaka-u. ac. jp/

Osaka University 


\title{
Replacement-Free Mass-Amplified Sandwich Assay with 180-MHz Electrodeless Quartz-Crystal Microbalance Biosensor
}

\author{
Hirotsugu Ogi ${ }^{a, b}$, Taiji Yanagida ${ }^{a}$, Masahiko Hirao $^{a}$, Masayoshi Nishiyama $^{c}$ \\ ${ }^{a}$ Graduate School of Engineering Science, Osaka University \\ Machikaneyama 1-3, Toyonaka, Osaka 560-8531, Japan \\ ${ }^{b}$ PRESTO, JST, 4-1-8 Honcho, Kawaguchi, Saitama, Japan \\ ${ }^{c}$ Central Workshop, Osaka University
}

Machikaneyama 1-2, Toyonaka, Osaka 560-0043, Japan

\begin{abstract}
This study presents the sensitivity-amplified detection method in the replacementfree electrodeless quartz crystal microbalance (QCM) biosensor. A sandwich assay is proposed for detecting $\mathrm{C}$ reactive proteins (CRP), where the biotinated second anti-CRP antibody is weighted by streptavidin for the sensitivity amplification. Because the first CRP antibody was immobilized nonspecifically on naked quartz surfaces, the sandwich assay was repeated using the same sensor chip, making the replacement-free assay possible. The mass-amplified sandwich assay detected the CRP solution of $0.1 \mathrm{ng} / \mathrm{ml}$. A methodology for determining the molecular mass of the injected protein is also proposed.
\end{abstract}

Key words: Electrodeless QCM, Replacement Free, Sensitivity Amplification, CRP 


\section{Introduction}

The quartz crystal microbalance (QCM) biosensor is mass-sensitive biosensor, and it allows detection of biomolecules captured by the corresponding receptor proteins immobilized on the oscillator surface through the change in the resonance frequency. The resonance-frequency change is principally caused by the mass loading effect ( Sauerbrey (1959)). The viscosity effect ( Kanazawa and Gordon (1985); Martin et al. (1991)) and the water-mass effect ( Höök et al. (1998); Reimhult et al. (2004)) contribute to lower the resonance fre-

quency as well, but their influence becomes insignificant at high frequencies ( Ogi et al. (2009a)). The mass sensitivity of QCM biosensor improves as the fundamental resonance frequency increases, because high-frequency QCMs use thinner quartz plate, enhancing the captured mass. Therefore, developing a higher-frequency QCM biosensor remains central issue in the biosensor field ( Uttenthaler et al. (2001); Cooper and Singleton (2007); Natesan et al. (2009); Furusawa et al. (2009)). We have proposed a wireless-electrodeless (WE) technique using an isolated quartz crystal and increased the fundamental frequency up to $170 \mathrm{MHz}$ ( Ogi et al. (2009a)).

As an important practical use, QCM biosensors are expected to detect biomarkers. However, human serum usually contains many blood proteins, and they are adsorbed on the sensor chip surfaces nonspecifically, making it difficult to extract the tiny frequency change caused by the binding of a target protein with the receptor on the surface from the total frequency response. Also, threshold concentrations are usually smaller than the detection limit of QCM biosensors. For overcoming this difficulty, we propose a mass-amplified sandwich assay using biotinated antibody, whose mass can be increased effectively by attaching 
streptavidin owing to extremely high affinity between biotin and streptavidin. As a target protein, we select the C-reactive protein (CRP), which is an important biomarker for checking inflammation. Its threshold level is about 30 $\mathrm{ng} / \mathrm{ml}$, and very quick assay is often required in an emergency case to judge the cause of illness. Thus, the WE-QCM can be a candidate because of its reusability and high sensitivity.

In the previous studies, gold nano particles have been used for the mass amplification in QCM assays ( Mao et al. (2006); Zhu et al. (2009); Chen et al. (2010); Pinto et al. (2010)). However, high affinity of gold with proteins ( Ogi et al. $(2009 b))$ causes nonspecific adsorption on receptor proteins. Also, preparation of surface modified particles takes longer time. Instead of the gold nano particles, we propose the mass amplification method using streptavidin, which quickly combines with biotin with extremely high affinity. Our mass-amplified sandwich assay detects CPR with concentration of 100 pg/ml. Furthermore, we propose a useful methodology to determine molecular mass of an injected ligand and apply it to the mass-amplified second anitbody.

\section{Experimental Section}

The first and second anti-CRP antibodies were obtained from Bethyl Laboratories, Inc. (No. A80-125A and No. A80-125F, respectively). CRP was obtained from Acris Antibodies GmbH (No. BA1087). The $\mathrm{NH}_{2}$-reactive biotin was from Dojindo (biotin labeling Kit- $\mathrm{NH}_{2}$ ). Streptavidin was from IBA Headquarters (No. 2-0203-001).

The proposed assay takes three steps. First, the first anti-CRP antibody is 
immobilized on the quartz surfaces nonspecifically. Second, the analyte (CRP solution) is injected. Third, the mass-amplified second anti-CRP-antibody solution is injected after the injection of the carrier solution for washing the surfaces. The schematic diagram of the sandwich assay is shown in Fig. 1(a).

A rectangular 9- $\mu \mathrm{m}$ thick AT-cut bare quartz $(2.5 \mathrm{~mm} \times 1.7 \mathrm{~mm})$, whose fundamental resonance frequency $f$ is near $182 \mathrm{MHz}$, was located at middle part of the flow channel by lightly sandwiching its corner edge by 1-mm thick siliconrubber gaskets. The carrier solution was phosphate buffered saline (PBS) solution ( $\mathrm{pH}$ 7.4), and nearly entire both surfaces of the quartz plate were exposed to the flowing solution. The two straight line antennas and a copper foil for grounding were embedded in bottom and top covers, respectively ( Ogi et al. (2009a)). The volume of the flow channel in the sensor cell is $2 \times 3 \times 40 \mathrm{~mm}^{3}$. The flow-injection analysis system used was homebuilt ( Ogi et al. (2007)).

The tone burst signal was applied to the generation antenna to excite the shear vibration, and the reverberating signals of the quartz plate were received by the detection antenna with noncontacting, which were processed by a superheterodyne phase sensitive detector. The resonance frequency of the quartz was determined from the phase of the received signal. Details of the signal processing can be found elsewhere ( Ogi et al. (2009c)).

The quartz crystal was first cleaned in a piranha solution $\left(98 \% \mathrm{H}_{2} \mathrm{SO}_{4}: 33 \% \mathrm{H}_{2} \mathrm{O}_{2}=\right.$ 7:3). After rinsing with ultrapure water, it was immersed in $500 \mu \mathrm{g} / \mathrm{ml}$ first anti-CRP-antibody solution for $5 \mathrm{~h}$ at $4{ }^{\circ} \mathrm{C}$ and rinsed by the PBS solution. Because the naked quartz surface adsorbs immunoglobulin G ( Ogi et al. (2009b)), it is possible to immobilize the first antibody just by immersing the cleaned sensor chip in the antibody solution. 
We prepared the mass-amplified second anti-CRP antibody as follows: The second antibody dissolved in the buffer solution was centrifuged in the filtration tube for 10 min with $8000 \mathrm{~g}$ for removing smaller foreign substances. The $\mathrm{NH}_{2}$-reactive biotin (biotin with active ester group at its end) was dissolved with $10 \mu$ l dimethyl sulfoxide (DMSO) and poured into the filtration tube together with a $100 \mu \mathrm{l}$ reaction buffer and incubated for $30 \mathrm{~min}$ at $37^{\circ} \mathrm{C}$. Then, the buffer solution was added in the tube and the solution was centrifuged to remove unreacted biotin molecules. This procedure was repeated three times, and we obtained the biotinated second anti-CRP antibody solution. The possible maximum concentration of the solution is $1 \mu \mathrm{g} / \mathrm{ml}$ estimated from the concentration of initial antibody solution, but this value could be lower because some second-antibody molecules were taken in the filter and uncollected.

Then, we mixed streptavidin in the second anti-CRP antibody solution, where concentrations of streptavidin was $1 \mu \mathrm{g} / \mathrm{ml}$. This solution was incubated for $30 \mathrm{~min}$ at $37^{\circ} \mathrm{C}$ before the injection.

CRP solutions $(0.1,1,10$, and $100 \mathrm{ng} / \mathrm{ml}$, in PBS) were prepared and injected in the reaction cell containing first antibody adsorbed on the quartz plate. The PBS solution was injected for a while for washing the sensor surfaces, and then the mass-amplified second antibody solution was injected.

\section{Results}

Figure 2 shows an example of the enhanced frequency change by the massamplified second-antibody solution. Injection of the $1 \mathrm{ng} / \mathrm{ml}$ CRP solution caused only 200-Hz frequency decrease, and the baseline increased after 15 
min from the injection. Thus, it would have been difficult to estimate the amount of the frequency change due to the binding of CRP with first antibody. However, the injection of the mass-amplified second-antibody solution caused nearly $5-\mathrm{kHz}$ frequency change, demonstrating the successful amplification of the mass sensitivity.

Figure 3(a) shows the binding curves observed after the arrival of the massamplified second-antibody solution, which were followed by injection of CRP solutions and the PBS solution. We observed the frequency decrease even for the injection of the second-antibody solution after the analyte solution without CRP molecules (0 ng/ml), indicating the nonspecific binding of the second antibody onto the first antibody. However, this frequency change was significantly smaller than those observed for the analyte solutions containing CRP molecules. The inset in Fig. 3(a) shows the correlation between the frequency decrement at $20 \mathrm{~min}$ from the arrival time of the mass-amplified second-antibody solution and the concentration of the CRP solution. However, such a correlation was obtained when we used antibodies from the same production lots, and the frequency change fluctuated among the assay with those from different lots as shown in Fig. 3(b).

\section{Discussion}

First, we emphasize that the electrodeless QCM is the replacement-free biosensor and the sensor chip was reusable after an assay by washing it with the piranha solution and rinsing with ultrapure water. This is an important advantage in treating many analyte solutions because and is only achieved by the electrodeless technique. The assay time $(\sim 20 \mathrm{~min}$ from the injection of the 
mass amplified solution) is shorter than those in conventional enzyme-linked immunosorbent assays ( $>\sim 3 \mathrm{~h})$.

Figure 2 clearly demonstrates that the mass-amplified second antibody increases the amount of the frequency change. We here propose a useful method to estimate molecular mass of injected ligand from a single frequency-monitoring measurement such as Fig. 2. This is made possible with the benefit of the highfrequency WE-QCM because of its high sensitivity to the added mass added on the sensor surfaces. Consider an equilibrium binding reaction between proteins $A$ and $B$ :

$$
A+B \Longleftrightarrow A B
$$

Where $A$ and $B$ denote the injected protein and the receptor protein on the quartz surfaces, respectively, and $A B$ denotes their complex on the surface (Figure 1(a) illustrates the case between the mass amplified antibody for $A$ and the CRP on the surface for B.) Assuming the pseudo-first-order reaction, the frequency change during the binding reaction $(A+B \longrightarrow A B)$ and that during the dissociating reaction $(A+B \longleftarrow A B)$ obey exponential functions with exponential coefficients $\alpha_{1}$ and $\alpha_{2}$, respectively ( Liu et al. (2003)). (In Fig. 2, we showed fitted exponential functions to extract the coefficients with red lines.) These coefficients relate with the reaction-velocity constants as

$$
\begin{array}{r}
\alpha_{1}=C_{A} k_{a}+k_{d}, \\
\alpha_{2}=k_{d} .
\end{array}
$$

Here, we denote $k_{a}$ and $k_{d}$ as the reaction velocity constants for association and dissociation reactions, respectively, and $C_{A}$ denotes the bulk concentration of molecule $A$. Therefore, the equilibrium constant $K_{A}=k_{a} / k_{d}$ can be 
determined by

$$
K_{A}=\frac{1}{C_{A}}\left(\frac{\alpha_{1}}{\alpha_{2}}-1\right)
$$

At the equilibrium state, the area molecular density of the complex $A B, C_{A B}$, can be expressed by ( Ogi et al. (2009b))

$$
C_{A B}=\frac{K_{A} C_{A} C_{B}^{0}}{K_{A} C_{A}+1}
$$

$C_{B}^{0}$ denotes the area molecular density of the receptor protein on the surface, and based on the Sauerbrey equation, it can be expressed by the frequency change $\Delta f_{B}$ observed when the receptor protein $B$ is injected (see Fig. 1(b)) as

$$
C_{B}^{0}=\frac{1}{2} \frac{\rho_{q} t_{q}}{M_{w}^{B}} \frac{\Delta f_{B}}{f}
$$

Where $\rho_{q}$ and $t_{q}$ are mass density and thickness of the quartz plate, respectively, and $M_{w}^{B}$ denotes the molecular mass of protein $B$. The factor $\frac{1}{2}$ appears because both surfaces can be the sensing region in our QCM. Considering that $C_{A B}$ is equal to the area molecular density of captured protein $A$ on surfaces, and it can be obtained from the frequency change $\Delta f_{A}$ like as $C_{B}^{0}$, we can determine the molecular mass of protein $A, M_{w}^{A}$ from equations (4) to (6) as

$$
M_{w}^{A}=M_{w}^{B}\left(\frac{\Delta f_{A}}{\Delta f_{B}}\right)\left(1-\frac{\alpha_{2}}{\alpha_{1}}\right)^{-1}
$$

This is a useful equation because we can determine the molecular mass of the injected protein from a single frequency measurement, and this can be made possible when the frequency of the QCM is so high that the simple Sauerbrey equation applies. We confirmed this equation using staphylococcus aureus protein $\mathrm{A}$ as the receptor protein $(B)$ and human immunoglobulin $\mathrm{G}$ 
(hIgG) as the analyte $(A)$, and obtained the predicted molecular mass of hIgG between 143 and $160 \mathrm{kDa}$, close to the molecular mass of hIgG of $150 \mathrm{kDa}$. (Because we published many QCM measurements on this binding system ( Ogi et al. (2009a, 2007, 2008)), we omit showing these experimental data.)

Using this theory, we estimate the molecular mass of the mass amplified second anti-CRP antibody. For example, we observe $\Delta f_{B}=\sim 200 \mathrm{~Hz}$ with the injection of the $1 \mathrm{ng} / \mathrm{ml}$ CPR solution and $\Delta f_{A}=\sim 4.6 \mathrm{kHz}$ caused by injection of the mass-amplified second-antibody solution in Fig. 2, yielding the molecular mass of the mass-amplified second antibody to be about 3,200 $\mathrm{kDa}$ with the molecular mass of CPR of $105 \mathrm{kDa}$. This value is much larger than the molecular mass of the unmodified second antibody ( $150 \mathrm{kDa})$ by a factor of 20. Therefore, mass amplification can be enhanced significantly. Because the IgG based antibody possesses several amino group terminals, a single biotinated antibody can bind with several streptavidin molecules (molecular mass: $53 \mathrm{kDa}$ ). Furthermore, because a single streptavidin has four binding sites with biotin, it can connect more than two antibody molecules through biotin terminals. The molecular mass of $3,200 \mathrm{kDa}$ is an averaged value, and we cannot identify the structure of the second-antibody complex. (It corresponds to a complex of six second antibodies with seven streptavidins attached to each antibody, for example.) The size will be affected by the incubation time and will affect the affinity between second antibody and CPR. Thus, it will be needed to control the incubation time in preparing the second-antibody solution to achieve the most suitable assay condition in our future study.

Finally, we discuss about the sensitivity on the CRP detection. We see a good correlation between the $\mathrm{CRP}$ concentration and the frequency decrement in Fig. 3(a), suggesting the applicability of the proposed sandwich method to di- 
agnosis with marker proteins. Such a correlation was obtained when we used the substances form the same sample lots, but the fluctuation of the frequency response becomes worse among measurements for different sample lots (Fig. 3(b)). Even in this case, the proposed assay can detect a low concentration CRP solution $(100 \mathrm{pg} / \mathrm{ml})$, for which the direct assay without second antibody failed to detect, confirming the high sensitivity of this assay. In this study, we used polyclonal antibodies, and their binding affinity and epitopes on CRP usually depend on the production lot. Also, the preparation of the mass-amplified second antibody requires high-affinity binding reactions, and the reaction time and temperature should significantly affect size and affinity of the second antibody. Thus, stable productions of individual substances with monoclonal antibodies will be needed for reliable diagnosis, and we will investigate systematically the sensitivity and reproducibility of this assay by changing time and temperature in preparing the mass-amplified second antibody with monoclonal antibodies.

\section{Conclusions}

We proposed mass-sensitivity amplification method using purely protein substances for the wireless-electrodeless QCM with a fundamental resonance frequency of $182 \mathrm{MHz}$. The sandwich assay was performed for detecting CRP. The first antibody was first immobilized nonspecifically, and the mass-amplified second antibody was injected, following the injection of the CRP solution. The detection was possible even for a smaller concentration CRP solution with $0.1 \mathrm{ng} / \mathrm{ml}$. From the binding curves and the presented theory, the molecular mass of the mass-amplified second antibody was estimated to be $3,200 \mathrm{kDa}$. 
The frequency response was fluctuated among measurements with substances from different production lots, and further improvements for purification of substances with monoclonal antibodies and strict controlling on time and temperature on preparation of the mass-amplified second antibody is needed for a reliable diagnosis use, which will be achieved in near future. 


\section{References}

Chen, Q.; Tang, W.; Wang, D.; Wu, X.; Li, N.; and Liu, F. Biosens. Bioelectron. 2010, 26, 575-579.

Cooper, M. A.; Singleton, V. T. J. Mol. Recognit. 2007, 20, 154-184.

Furusawa, H.; Komatsu, M.; Okahata, Y. Anal. Chem. 2009, 81, 1841-1847.

Höök, F.; Rodahl, M.; Brzezinski, P.; Kasemo, B. Langmuir 1998, 14, 729-734.

Kanazawa, K. K.; Gordon, J. G. Anal. Chim. Acta. 1985, 175, 99-105.

Liu, Y.; Yu, X.; Zhao, R.; Shangguan, D.; Bo, Z.; Liu, G. Biosens. Bioelectron. 2003, 19, 9-19.

Mao, X.; Yang, L.; Su, X.-L.; and Li, Y. Biosens. Bioelectron. 2006, 21, 11781185.

Martin, S. J.; Granstaff, V. E.; Frye, G. C. Anal. Chem. 1991, 63, 2272-2281.

Natesan, M.; Cooper, M. A.;Tran, J. P;Rivera, V. R.;Poli, M. A. Anal. Chem. 2009, 81, 3896-3902.

Ogi, H.; Motohisa, M.; Hatanaka, K.; Ohmori, T.; Hirao, M.; Nishiyama, M. Biosens. Bioelectron. 2007, 22, 3238-3242.

Ogi, H.; Fukunishi, Y.; Omori, T.; Hatanaka, K.; Hirao, M.; Nishiyama, M. Anal. Chem. 2008, 80, 5494-5500.

Ogi, H.; Nagai, H,; Fukunishi, Y.; Hirao, M.; Nishiyama, M.; Anal. Chem. 2009, 81, 8068-8073.

Ogi, H.; Fukunishi, Y.; Nagai, H.; Okamoto, K.; Hirao, M.; Nishiyama, M. Biosens. Bioelectron. 2009, 24, 3148-3152.

Ogi, H.; Okamoto, K.; Nagai, H.; Fukunishi, Y.; Hirao, M. Anal. Chem. 2009, 81, 4015-4020.

Pinto, E. M.; Barsan, M. M.; and Brett, C. M. A. J. Phys. Chem. B. 2010, $114,15354-15361$. 
Reimhult, E.; Larsson, C.; Kasemo, B.; Höök, F. Anal. Chem. 2004, 76, 72117220.

Sauerbrey, G. Z. Phys. 1959, 155, 206-222.

Uttenthaler, E.; Schr'aml, M.;Mandel, J.; Drost, S. Biosens. Bioelectron. 2001, $16,735-743$.

Zhu, Z.; Su, Y.; Li, J.; Li, D.; Zhang, J.; Song, S.; Zhao, Y.; Li, G.; and Fan, C. Anal. Chem. 2009, 81, 7660-7666. 


\section{Figure Captions}

Fig. 1 (Color)(a)Schematic of the sandwich assay using the mass-amplified second anti-CRP antibody. A, B, and AB denote the mass-amplified second antibody, the CRP adsorbed on the first antibody, and their complex, respectively. (Note that actual numbers of second antibody and streptavidin are larger than those in this schematic.) (b)Schematic of a frequency response during the immobilization, binding, and dissociating reactions.

Fig. 2 (Color)Frequency response for injections of a $1 \mathrm{ng} / \mathrm{ml} \mathrm{CRP}, \mathrm{PBS}$, and the mass-amplified second-antibody solutions. Arrows indicate the arrival time of the solutions at the sensor cell.

Fig. 3 (a) Binding curves observed for the injection of the mass-amplified second-antibody solution after the injection of the CRP solution with various concentrations. Arrows indicates arrival of the PBS solution. The numbers denote the concentration of the CRP solution injected before the massamplified second-antibody solution. The inset shows the correlation between the frequency change and the $\mathrm{CRP}$ concentration $C_{C R P}$ at $20 \mathrm{~min}$. (b)Frequency decrement and CRP concentration $C_{C R P}$ at 20 min for measurements with antibodies from different production lots. 


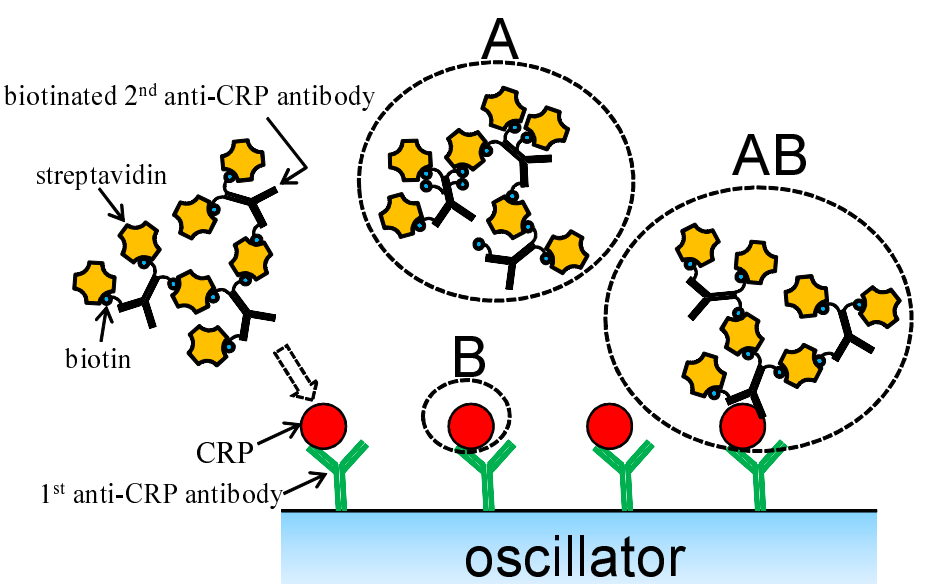

(a)

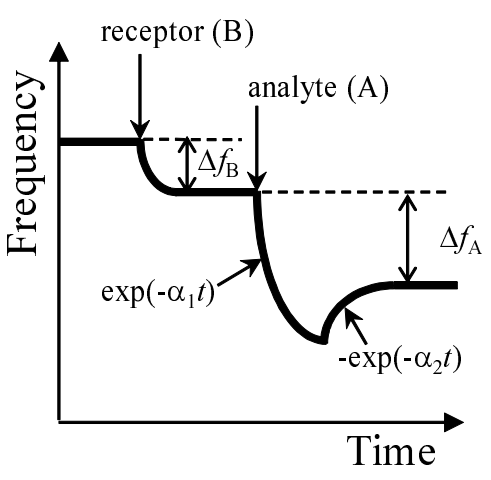

(b)

Fig. 1. 


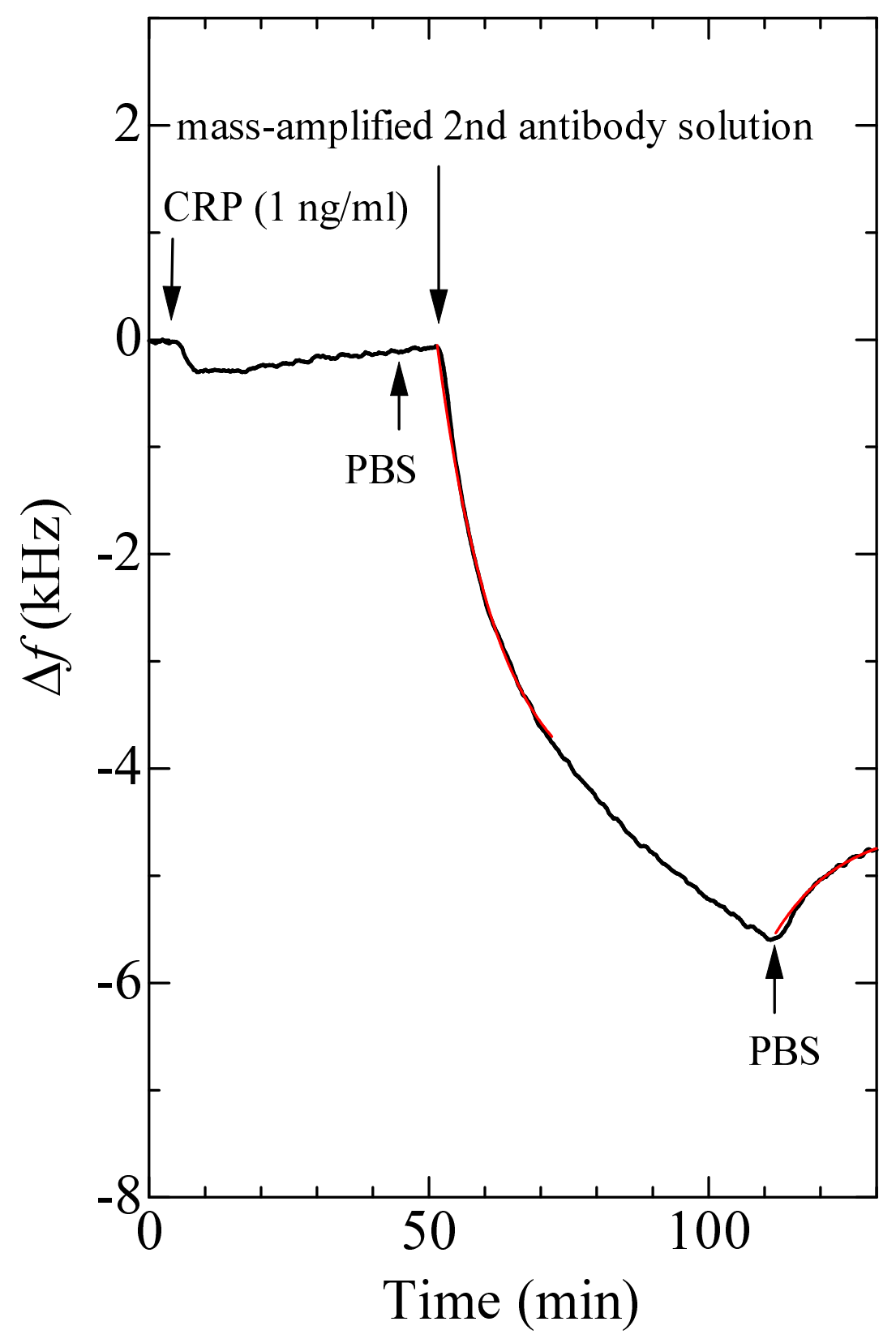

Fig. 2. 

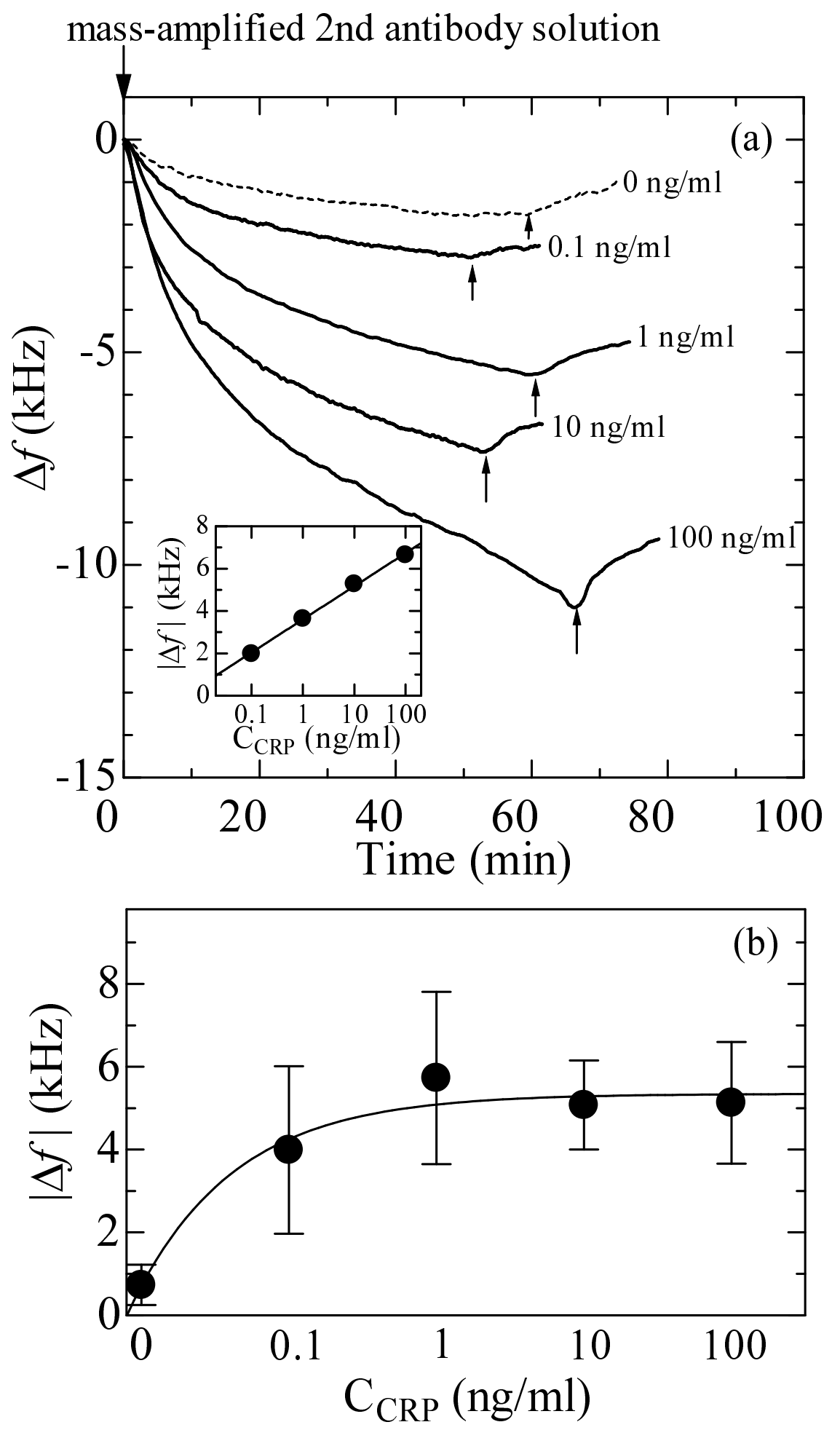

Fig. 3. 